\title{
Tandem plasmapheresis and continuous kidney replacement treatment in pediatric patients
}

\author{
Naile Tufan Pekkucuksen ${ }^{1,2}$ (D) Katie E. Sigler ${ }^{2} \cdot$ Ayse Akcan Arikan $^{2,3} \cdot$ Poyyapakkam Srivaths $^{2}$
}

Received: 9 September 2019 / Revised: 15 April 2020 / Accepted: 27 April 2020 / Published online: 27 October 2020

(C) IPNA 2020

\begin{abstract}
Background The objectives of the study are to describe tandem therapeutic plasma exchange (TPE) and continuous kidney replacement therapy (CKRT) patients' outcomes in a large institution.

Methods We reviewed pediatric patients receiving tandem TPE and CKRT from 2013 to 2016. Over the study period, 63 discrete patients received tandem TPE and CKRT for a total of 378 TPE procedures on 1676 days on CKRT.

Results Patient age ranged from newborn to 19 years old with weights ranging from 2.31 to $112.3 \mathrm{~kg}$ (17 patients were $<10 \mathrm{~kg}$ and less than 1 year old). All procedures were completed in intensive care units (ICU) as CKRT can only be done in this environment. All treatments completed successfully; majority of patients (90\%) developed hypocalcemia though none were symptomatic. Case mortality rate was 40\%. Disease severity scores at ICU admission were higher and time to TPE and CKRT start was longer in the deceased group. Conclusions As a conclusion, though complications including hypocalcemia are common with tandem TPE and CKRT in pediatrics, patients remained asymptomatic. Such treatments have to be carefully planned with interdisciplinary teams to address indications, technicalities, and complications.
\end{abstract}

Keywords Tandem $\cdot$ Plasmaphresis $\cdot$ Plasma exchange $\cdot$ CKRT $\cdot$ Pediatrics $\cdot$ PICU

\section{Introduction}

Plasmapheresis (PP) is a blood purification procedure, based on the extracorporeal separation of blood cellular components from colloid plasma and return of the cells to the patient circulatory system combined with a replacement solution of either $5 \%$ albumin or donor fresh frozen plasma (FFP). It has been used successfully to reduce the concentration of large molecules such as pathogenic antibodies, immune complexes, cryoglobulins, and lipoproteins [1]. Therapeutic plasma exchange (TPE) indications are categorized by the American Society for Apheresis (ASFA) [2]. Continuous kidney replacement therapy (CKRT) is the treatment modality for acute kidney injury (AKI), fluid

Naile Tufan Pekkucuksen

nailetufan@gmail.com

1 Department of Pediatrics, Pediatric Nephrology Division, University of Florida, Gainesville, FL, USA

2 Department of Pediatrics, Renal Section, Baylor College of Medicine, Houston, TX, USA

3 Department of Pediatrics, Section of Critical Care Medicine Texas Children's Hospital, Baylor College of Medicine, Houston, TX, USA overload (FO), and electrolyte and metabolic imbalance for unstable patients. In some situations, patients need multiple modalities of extracorporeal support. Simultaneous use of multiple modalities with dedicated machines combined in series or in parallel is defined as tandem therapies (Fig. 1). Patients who have received tandem TPE with intermittent hemodialysis (HD) in both adults and children have been described [3-11]. Yorgin et al. described the use of plasmapheresis during CKRT in 2000 [12]. However, there is infrequent reported experience about the simultaneous use of TPE and CKRT (here forth referred to as tandem TPE) in pediatric patients.

In this retrospective study, we aim to describe clinical characteristics and patient outcomes for tandem TPE with CKRT in critically ill children and investigate the efficacy or safety. We hypothesized that clinically significant hypocalcemia during tandem TPE and CKRT can be medically managed to allow safe completion of TPE procedure.

\section{Methods}

The study was approved by the Baylor College of Medicine Institutional Review Board (IRB). This was a retrospective, 


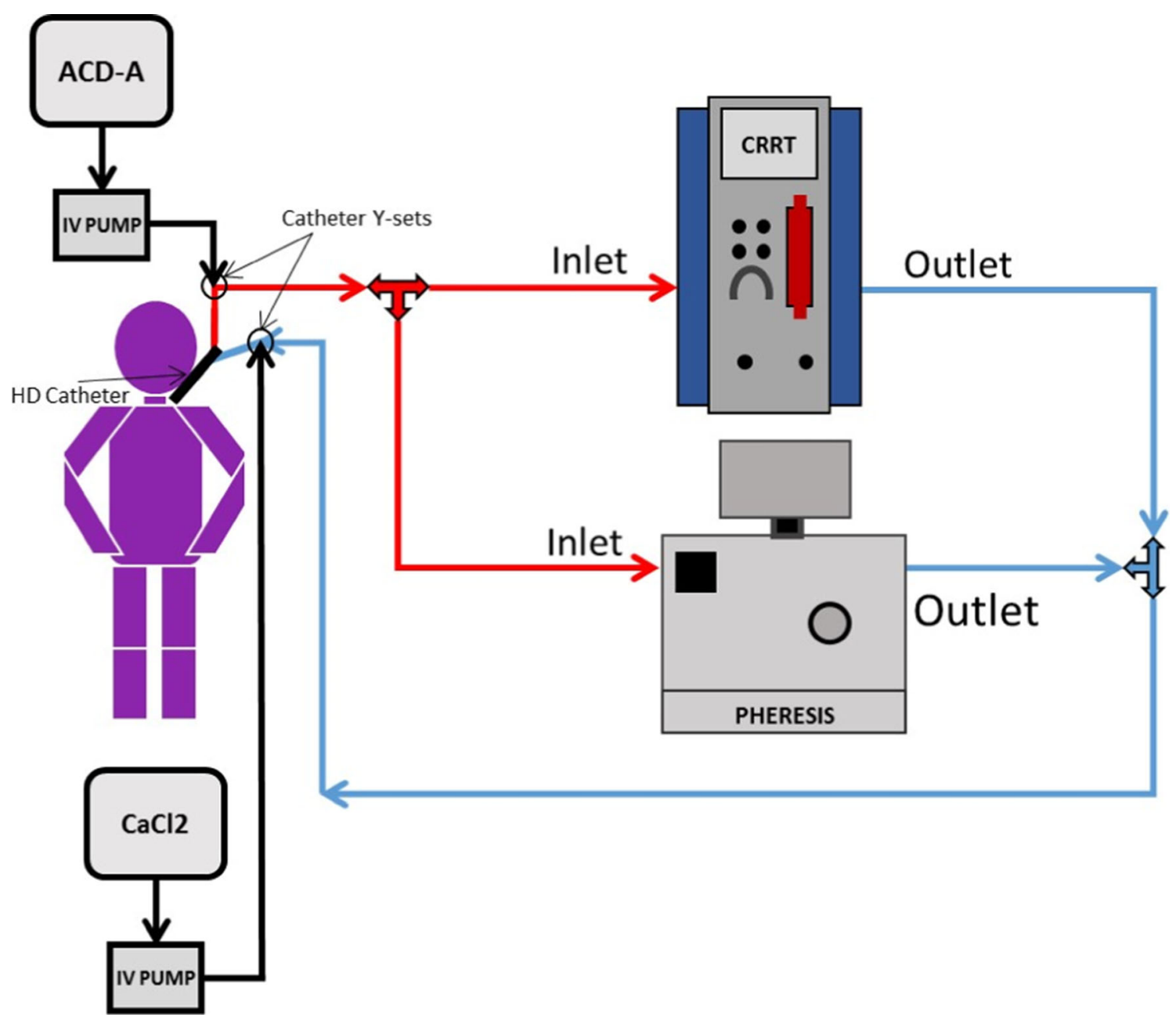

Fig. 1 Depicting the tandem parallel circuit in our institution

single-centered descriptive study. All demographic, clinical, laboratory, TPE and CKRT data were extracted with clinical information from patient medical records in 2013-2016. Pediatric risk of mortality 2 (PRISM2) was calculated at PICU admission as a disease severity score, and vasoactiveinotropic scores (VIS) and pediatric logistic organ dysfunction (PELOD) scores were calculated on CKRT initiation day for each patient. For chronic liver failure patients, CLIF-SOFA scores were calculated at ICU admission. Mortality was defined as patient death before discharge. The decision to start TPE and CKRT was made by the consultant renal physician based on institutional protocols. All patients who received tandem TPE and CRKT were included in the study.

A double lumen hemodialysis catheter was used for extracorporeal therapy access except for patients on ECMO, where the TPE and CKRT machines were connected to the ECMO circuit.

In our institution, centrifugal-based TPE is performed using Spectra Optia ${ }^{\circledR}$ (Terumo BCT, Lakewood, CO); as per institutional protocol, all CKRT patients receive hemodiafiltration (CVVHDF) at minimum starting dose of
$2000 \mathrm{ml} / 1.73 \mathrm{~m}^{2} / \mathrm{h}$ with Baxter® Prismasol replacement solutions without calcium. If a patient was on ECMO, calciumbased replacement solution was chosen due to non-citratebased anticoagulation. Blood flow rate (BFR) of the CKRT was prescribed as 3-6 ml $/ \mathrm{min} / \mathrm{kg}$. The BFR of TPE was determined by the machine depending upon the replacement fluid but is approximately $2 \mathrm{ml} / \mathrm{min} / \mathrm{kg}$. CVVHDF was prescribed with prefilter dilution and regional citrate anticoagulation, and filtration fraction was kept below $25 \%$. All patients also received calcium chloride or/and calcium gluconate infusions either into the return line of CKRT circuit or via separate central line. Calcium solution was chosen based purely on availability. Starting citrate (ACD-A, containing $112 \mathrm{mmol} / \mathrm{L}$ of citrate anion) and calcium chloride infusions $(2.16 \mathrm{mg} / \mathrm{mL}$ elemental calcium) were prescribed relative to blood flow (BFR; $1-1.5 \times($ as $\mathrm{ml} / \mathrm{h})$ and $0.6 \times \mathrm{BFR}(\mathrm{ml} /$ h), respectively) and titrated according to serum and circuit ionized calcium levels. The lower and upper limits of ionized calcium as reported by our clinical laboratory are less than $1.1 \mathrm{mmol}$ per liter and greater than $1.3 \mathrm{mmol}$ per liter, respectively. Targeted post CKRT filter calcium level was $0.3-0.5$ 
$\mathrm{mmol} / \mathrm{L}$. For tandem TPE, apheresis lines are connected via a three-way stop-cock to the CKRT circuit in parallel without using additional anticoagulation (Fig. 1). For patients on extracorporeal membrane oxygenation (ECMO), heparin is the most common anticoagulant used. We did not use separate anticoagulation for CKRT or TPE while on ECMO. Circuit connection schema is provided in Fig. 2.

The extracorporeal volume of the TPE machine is $240 \mathrm{ml}$. Red blood cells (RBC) were diluted to $35 \%$ hematocrit, then used to prime the circuit for patients of less than $25 \mathrm{~kg}$ body weight. For indications of thrombotic microangiopathy (TMA), thrombocytopenia-associated multi-organ failure (TAMOF), diffuse alveolar hemorrhage (DAH), thrombotic thrombocytopenic purpura (TTP), and liver failure (LF), FFP was the only replacement fluid used. For other indications such as antibody-mediated rejection, we used 5\% albumin.

\section{Statistical analysis}

Continuous data is presented as mean $\pm \mathrm{SD}$ or median (IQR) based on the distribution statistical results. Categorical data is presented as percentages. Shapiro-Wilk $W$ test was used to test the normality of distribution. Due to small study size, mostly median (IQR) is reported. Student $t$ test or $\chi^{2}$ test are used to compare the data as appropriate. To evaluate independence associations between survivors and non-survivors, logistic regression model was used to include both categorical variables, such as the presence of hypocalcemia, PP diagnostic categories, and presence of separate lock, and continuous variables such as age and PRISM score in the model. All statistical analyses were performed using Stata 15 (Stata, Inc, College Station, Texas). $P$ value less than 0.05 was accepted as significant.

\section{Results}

Tandem TPE and CKRT were prescribed for various conditions including TAMOF, TMA, DAH, and TTP. From 2013 to 2016, 63 patients (33 female (52\%) and 30 male (48\%)) received tandem TPE and CKRT. Mean patient age was $7 \pm$ 6.47 (median age was 5 IQR $1 ; 13$ ) years and mean weight was $31.79 \pm 28.31 \mathrm{~kg}$ (median: $17.8 \mathrm{~kg}$ IQR $10 ; 54$ ). Seventeen patients (27\%) were under 1 year old and less than $10 \mathrm{~kg}$. Primary diagnosis distribution was as follows: fulminant liver failure (33\%), hematology/oncology (15\%), infectious/sepsis $(15 \%)$, cardiac failure (12\%), metabolic disorders $(9 \%)$, rheumatologic disease $(7 \%)$, and primary kidney disease (4\%). Most common ICU admission reason was shock [23/63(36.5\%)]. Other common admission reasons were 13 (21\%) fulminant liver failure and $8(13 \%)$ cardiac failure. The remaining patients were admitted due to less common reasons like electrolyte imbalance or bleeding. Median length of hospital stay was 54 days (IQR 22; 97); median ICU stay

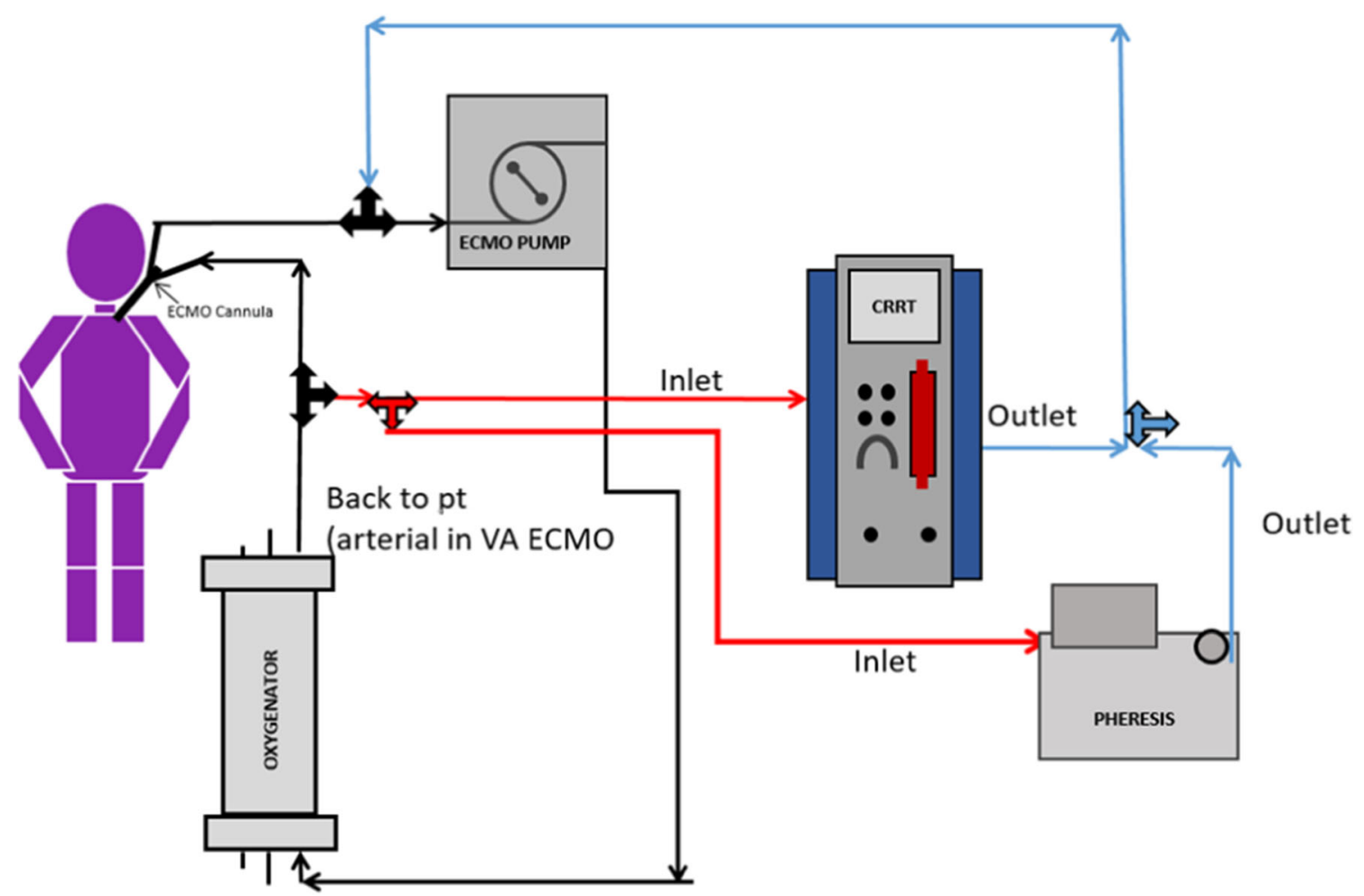

Fig. 2 Triple tandem treatment: ECMO-PP-CKRT 
was 29 days (IQR 13; 55). During the ICU course, 62 patients (98\%) received invasive mechanical ventilation support. Median invasive mechanical ventilation length was 18 days (IQR 8; 47). Twenty-six (41\%) patients needed highfrequency oscillation (HFOV) for severe respiratory failure. Median days on HFOV were 4 (IQR 2; 7). Forty-eight out of 63 patients $(76 \%)$ needed inotropic support due to severe cardiovascular instability. Twenty patients (32\%) received one, 18 patients (29\%) received two, and 10 patients (16\%) received three different inotropic agents at CKRT initiation.

A total of 378 tandem TPE sessions were performed during the study duration. Median number of sessions per patient was 5 (IQR 3; 7). Median number of days from ICU admission to TPE initiation was 2 (IQR 1; 6). Twenty (32\%) patients received TPE due to coagulopathy and hepatic encephalopathy caused by acute or acute on chronic liver failure. Nineteen (30\%) patients received TPE due to thrombocytopeniaassociated multi-organ failure (TAMOF). Five patients (7\%) received TPE due to thrombotic microangiopathy (TMA). When indications for TPE were grouped as per ASFA category, 57 patients (90\%) had ASFA category 3, 2 patients (3\%) had category 2 , and 4 patients $(6 \%)$ had category 1 indication. Fresh frozen plasma (FFP) was used for replacement for $95 \%$ of the patients and albumin 5\% solution was used for 2 patients. One patient received both replacements but in different sessions.

The leading CKRT indication was acute kidney injury (AKI) and fluid overload (FO) in $65 \%$ of patients. Other indications were hyperammonemia and hepatic encephalopathy in $24 \%$ and chronic kidney disease needing kidney replacement therapy in $11 \%$. Median FO at CKRT start was 15\% (IQR 6; 25). Median number of days from ICU admission to CKRT initiation was 1 (IQR 1; 4). Median number of days on CKRT was 12 (IQR 6; 37).

Some patients needed extracorporeal membranous oxygenation (ECMO) along with TPE and CKRT. Fifteen patients received ECMO. Median number of days on ECMO was 13 (IQR 6; 27).

Ninety percent of patients had hypocalcemia and $93 \%$ of patients had hypercalcemia at least once during the treatments. A nested analysis of 36 patients (out of a total of 63 patients) for whom all the treatment details were available was performed; 147 instances of hypocalcemia $<1 \mathrm{mmol} / \mathrm{L}$ and 13 instances of hypercalcemia $>1.5 \mathrm{mmol} / \mathrm{L}$ occurred over 203 treatments; the nadir ionized calcium was $0.7 \mathrm{mmol} / \mathrm{L}$ and the high was $1.8 \mathrm{mmol} / \mathrm{L}$. Twenty-seven patients had citrate accumulation as defined by a total calcium to ionized calcium ratio $(\mathrm{mmol} / \mathrm{L} / \mathrm{mmol} / \mathrm{L})$ of $>2.5$ during the treatments. None of these episodes was symptomatic. There was no significant difference between the occurrence of hypocalcemia and exchanged plasma volume, type of replacement fluid, or number of sessions; however, citrate accumulation occurrence was associated with the presence of liver disease $(p=0.02)$. No clotting was reported during the TPE sessions. Twenty-five patients $(40 \%)$ died at the end of the hospital course. Thirteen patients died due to multi-organ dysfunction (MODS), 8 due to withdrawal of life-sustaining therapies, and 4 due to cardiac failure (Table 1).

We compared the clinical features of survivors to non-survivors. There was no difference between the two groups in terms of demographics. There were no differences in length of treatment (days on CKRT and number of TPE sessions), length of ICU stay, or length of mechanical ventilation between the survivor and non-survivor groups. Receiving ECMO, HFOV, and inotrope support were not found to be associated with mortality. Time to CKRT and TPE initiation were longer in non-survivors $(3$ days $(1,9)$ vs. 1 day $(1,2)$ to CKRT, $p=0.02)$ and $(4$ days $(2,13)$ vs. 2 days $(1,3)$ TPE, $p=$ 0.007). Also, PRISM score at ICU admission of non-survivors was significantly higher than survivors (16.2 \pm 6.54 vs. $12.7 \pm$ $5.87, \mathrm{p}=0.031)$. Since time to initiation of CKRT and TPE were highly correlated, we used only time to TPE initiation in the logistic regression model which yielded both PRISM score and time to initiation as independently associated with mortality (Table 2).

We examined available platelet counts in the subset of TAMOF patients; since thrombocytopenia is a required condition for diagnosis, all 18 patients had platelet count on the day of procedure. $8 / 18$ patients also had platelet count available 3 days prior to initiation of TPE; 5 out of these 8 patients had low platelet count and 3 did not survive. Platelet counts improved in all patients with TTP while only in $60 \%$ of patients with TAMOF.

\section{Discussion}

Therapeutic plasma exchange is indicated for a variety of conditions that are classified by the ASFA [2]. Most non-renal ASFA class I and II diseases do not have a kidney insufficiency during the hospital course and do not need kidney replacement therapy [13]. In our study, we had only three patients with primary neurological diseases who needed kidney replacement therapy due to either AKI or FO. Our patient population had a wide variety of indications for tandem treatments. The 2 most frequent indications were acute liver failure $(20 / 63)$ and TAMOF (19/63). We have institution-based guidelines for the treatment of TAMOF and acute liver failure (Figs.1 and 2). Other studies have reported that solid organ transplantation and antibody-mediated organ rejection and TAMOF are the most common indications for tandem treatments $[9,11,12]$.

The first study in pediatrics that described tandem TPE with CKRT was in a 14-year-old patient with TTP-like process after Bacillus cereus sepsis following acute B-cell ALL induction therapy. She received continuous venovenous 
Table 1 Patient and treatment characteristics in study population

\begin{tabular}{|c|c|c|c|c|}
\hline & $\begin{array}{l}\text { All patients } \\
(n=63)\end{array}$ & $\begin{array}{l}\text { Survivor } \\
(n=38)\end{array}$ & $\begin{array}{l}\text { Non-survivor } \\
(n=25)\end{array}$ & $P$ values \\
\hline Gender no (female) & 33 female & 21 female & 12 female & 0.57 \\
\hline Age (years) (median (IQR)) & $6.47(1-13)$ & $8.5(1-13)$ & $2(0.75-10)$ & 0.3 \\
\hline Weight (kg) (median (IQR)) & $7.8(10-54)$ & $11.2(11.52-54)$ & $25(7.5-50)$ & 0.12 \\
\hline No of TPE sessions/patient (median (IQR)) & $5(3-7)$ & $5(5-7)$ & $4(2-6)$ & 0.06 \\
\hline Mean plasma volume exchanged & $1.34 \pm 0.21$ & $1.35 \pm 0.18$ & $1.32 \pm 0.25$ & 0.6 \\
\hline CKRT days (median (IQR)) & $12(6-37)$ & $13(6-39)$ & $12(7-24)$ & 0.7 \\
\hline PRISM score on admission (mean $\pm \mathrm{SD}$ ) & $14.09 \pm 6.33$ & $12.6 \pm 5.8$ & $16.16 \pm 6.54$ & 0.031 \\
\hline PELOD score at start of CKRT (median (IQR)) & $20(11-22)$ & $12(10.5-22)$ & $22(12-22)$ & 0.16 \\
\hline $\begin{array}{l}\text { Time to initiation of TPE from PICU } \\
\text { admission (days) (median (IQR)) }\end{array}$ & $2(1-6)$ & $2(1-3)$ & $4(2-13)$ & 0.029 \\
\hline $\begin{array}{l}\text { Time to initiation of CKRT from PICU } \\
\text { admission (days) (median (IQR)) }\end{array}$ & $1(1-4)$ & $1(1-2)$ & $3(1.9)$ & 0.02 \\
\hline $\operatorname{ECMO}(n: 15)$ days on ECMO (median, IQR) & $13(6-27)$ & $11(6-17)$ & $17(3-40)$ & \\
\hline
\end{tabular}

hemofiltration/hemodiafiltration (CVVHD) and tandem TPE. After combined therapy, her ventilator and neurological status improved significantly [12]. Another study discussed two cases who received 16 sessions in total of concurrent CKRT with plasmapheresis [1].

In our hospital, we performed 378 tandem TPE in 63 patients during the study period. All sessions were completed successfully. Using FFP replacement and performing tandem TPE pose a risk of hypocalcemia and we saw that in our patient population, with the majority of patients developing hypocalcemia due to citrate load from FFP. We also observed hypercalcemia in these patients which may be due to treatment of hypocalcemia episodes with higher calcium replacement solution. Since a third of our patients also had liver failure, we observed citrate accumulation in these patients; however, all these biochemical abnormalities were asymptomatic.

We found that the PRISM II score at ICU admission was significantly higher in the mortality group indicating a higher disease severity. The time to initiation of TPE was longer in the mortality group, which may be either due to more unstable patients in the mortality group or delayed recognition for need of treatment, since $62 \%$ of patients in TAMOF category fulfilled the platelet criteria 3 days before initiation of therapy.

These study findings related to mortality cannot be attributed as causal since this was a retrospective study, and

Table 2 Logistic regression for odds for survival

\begin{tabular}{llll}
\hline Variable & Odds ratio & $P$ value & $95 \%$ CI \\
\hline Age & 0.973 & 0.8 & $0.78-1.21$ \\
Time to TPE initiation & 0.86 & 0.029 & $0.76-0.98$ \\
PRISM score on ICU admission & 0.86 & 0.022 & $0.76-0.97$ \\
TPE indication & 1.06 & 0.6 & $0.82-1.38$ \\
\hline
\end{tabular}

generalizability could be limited since this was from a single institution.

Hypocalcemia was reported $22-25.7 \%$ and $5.3 \%$ separately during CKRT and TPE respectively [14-16]. In our study, 147 instances of hypocalcemia $<1 \mathrm{mmol} / \mathrm{L}$ occurred over 203 treatments (72\%) in 57 of $63(90 \%)$ patients. Adverse effects can be seen more often during tandem treatments because of treatment complexity and patients' unstable status.

\section{Conclusion}

The indications for tandem TPE with CKRT in pediatrics from our institution are much more for category 3 , signifying occurrence of AKI in multi-organ involvement in the ICU setting. Mortality reflects the severity of illness as expected but further studies are needed to evaluate effects of the treatment initiation time on mortality. Complications including hypocalcemia are common though patients remained asymptomatic and can be managed safely with increased calcium infusion rate and following ionized calcium levels closely. Such treatments need to be planned carefully with interdisciplinary teams to address indications, technicalities, and complications.

Acknowledgments We would like to thank Saliha Erdem (Baylor College of Medicine, Renal Section, Department of Pediatrics) and Dr Alyssa Riley and Ms. Gracious Sarasak for assistance with collection of data and for the tandem figures respectively.

\section{References}

1. Eding DM, Jelsma LR, Metz CJ, Steen VS, Wincek JM (2011) Innovative techniques to decrease blood exposure and minimize interruptions in pediatric continuous renal replacement therapy. Crit Care Nurse 31(1):64-71 
2. Schwartz J, Padmanabhan A, Aqui N, Balogun RA, ConnellySmith L, Delaney M et al (2016) Guidelines on the use of therapeutic apheresis in clinical practice-evidence-based approach from the writing committee of the American Society for Apheresis: The seventh special issue. J Clin Apher 31(3):149-162

3. Bhowmik D, Jain PK, Masih JA, Saha D, Gupta S, Agarwal SK et al (2001) Tandem plasmapheresis and hemodialysis. Ther Apher 5(5):439-441

4. Dechmann-Sultemeyer T, Linkeschova R, Lenzen K, Kuril Z, Grabensee B, Voiculescu A (2009) Tandem plasmapheresis and haemodialysis as a safe procedure in 82 patients with immunemediated disease. Nephrol Dial Transplant 24(1):252-257

5. Perez-Saez MJ, Toledo K, Ojeda R, Crespo R, Soriano S, Alvarez de Lara MA et al (2011) Tandem plasmapheresis and hemodialysis: efficacy and safety. Ren Fail 33(8):765-769

6. Paglialonga F, Ardissino G, Biasuzzi A, Testa S, Edefonti A (2012) Tandem plasma-exchange and haemodialysis in a paediatric dialysis unit. Pediatr Nephrol 27(3):493-495

7. Bridges BC, Hardison D, Pietsch J (2013) A case series of the successful use of ECMO, continuous renal replacement therapy, and plasma exchange for thrombocytopenia-associated multiple organ failure. J Pediatr Surg 48(5):1114-1117

8. Filler G, Clark WF, Huang SH (2014) Tandem hemodialysis and plasma exchange. Pediatr Nephrol 29(11):2077-2082

9. Dyer M, Neal MD, Rollins-Raval MA, Raval JS (2014) Simultaneous extracorporeal membrane oxygenation and therapeutic plasma exchange procedures are tolerable in both pediatric and adult patients. Transfusion 54(4):1158-1165
10. Ide K, Muguruma T, Shinohara M, Toida C, Enomoto Y, Matsumoto $S$ et al (2015) Continuous veno-venous hemodiafiltration and plasma exchange in infantile acute liver failure. Pediatr Crit Care Med 16(8):e268-e274

11. Sirignano RM, Paden ML, Fasano R, Meyer EK (2018) Epidemiology of therapeutic apheresis with a multidisciplinary approach at a high volume pediatric center. J Clin Apher 33(3):297302

12. Yorgin PD, Eklund DK, al-Uzri A, Whitesell L, Theodorou AA (2000) Concurrent centrifugation plasmapheresis and continuous venovenous hemodiafiltration. Pediatr Nephrol 14(1):18-21

13. Prytula A, Vande Walle J, Verhelst H, Eloot S, Claus S, De Jaeger A et al (2015) Therapeutic plasma exchange in children with acute autoimmune central nervous system disorders. Int J Artif Organs 38(9):494-500

14. Akhoundi A, Singh B, Vela M, Chaudhary S, Monaghan M, Wilson GA et al (2015) Incidence of adverse events during continuous renal replacement therapy. Blood Purif 39(4):333-339

15. Bianchi NA, Altarelli M, Eckert P, Schneider AG (2020) Complications of regional citrate anticoagulation for continuous renal replacement therapy: an observational study. Blood Purif 1-9

16. Sigler K, Lee J, Srivaths P (2018) Regional citrate anticoagulation with calcium replacement in pediatric apheresis. J Clin Apher 33(3):274-277

Publisher's note Springer Nature remains neutral with regard to jurisdictional claims in published maps and institutional affiliations. 